\title{
DETERMINACIÓN DE LA ACTIVIDAD FOTOCATALÍTICA DE ZnS Y CdS EN LA DEGRADACIÓN DE CIANUROS
}

\author{
LAURA GARCÍA, ISABEL SAÉNZ, IDALIA GÓMEZ
}

Laboratorio de Materiales I, Universidad Autónoma de Nuevo León, San Nicolás, México

\begin{abstract}
Resumen
El presente trabajo muestra los resultados del estudio de la actividad fotocatalítica del $\mathrm{ZnS}$ y CdS en la degradación de cianuros, evaluando los efectos de cambio en morfología, área superficial específica y estabilidad en el sistema. Los análisis por difracción de rayos X, espectroscopias de FTIR, UV-Vis y AA, así como los análisis texturales y microestructurales, indican que bajo las condiciones de radiación de $365 \mathrm{~nm}$ y concentraciones de hasta $20 \mathrm{ppm}$ de $\mathrm{CN}^{-}$el ZnS es más inestable que el CdS, presentando un proceso de aglomeración. Así mismo, el CdS muestra una mayor actividad fotocatalítica en la degradación de cianuros en una concentración de $20 \mathrm{ppm}$.
\end{abstract}

\section{Introducción}

Uno de los problemas con que cuenta la sociedad es el alto grado de contaminación en agua debido a la presencia de compuestos sumamente tóxicos con una alta resistencia a la degradación, entre los cuales se encuentran los cianuros.

Los métodos tradicionales de separación consisten básicamente en transferir la sustancia tóxica de una corriente a otra, por lo cual el problema persiste. Los métodos de remediación buscan por su parte degradar estas sustancias y transformarlas en otras no tóxicas o menos tóxicas.

Por lo que se han desarrollado nuevas tecnologías de purificación, que toman en cuenta la naturaleza y las propiedades fisicoquímicas de las aguas o efluentes a tratar [1].

Las Tecnologías Avanzadas de Oxidación (TAO's), pueden aplicarse a la remediación y destoxificación en pequeña o mediana escala, se basan en procesos fisicoquímicos capaces de producir cambios profundos en la estructura química de los contaminantes [2-6].

Dentro de las TAO's se encuentra la fotocatálisis heterogénea, cuyo principio se basa en la física de semiconductores, cuando es iluminado con fotones de energía igual o mayor que la energía de banda prohibida $\left(\mathrm{E}_{\mathrm{g}}\right)$. Siendo el $\mathrm{TiO}_{2}$ el compuesto más utilizado en la degradación de compuestos orgánicos [7-9]. El ZnS tiene una $\mathrm{E}_{\mathrm{g}}$ parecida al del $\mathrm{TiO}_{2}(3.2 \mathrm{eV})$, entonces se requiere longitudes de onda menores que $400 \mathrm{~nm}$ para su activación [10], lo contrario ocurre para el CdS ya que tiene una $\mathrm{E}_{\mathrm{g}}$ menor al del $\mathrm{TiO}_{2}$, por lo cual se puede activar con longitudes de onda incluso mayores de $400 \mathrm{~nm}$, es decir en la región del visible.

En este trabajo se presentan los resultados del estudio para la determinación de la actividad fotocatalítica del CdS y ZnS en la degradación de cianuros, esto bajo una radiación ultravioleta de $365 \mathrm{~nm}$. El CdS y ZnS utilizados se sintetizaron por el método de coprecipitación. También se muestran los cambios texturales y microtexturales que presentaron los semiconductores debido al sistema químico utilizado.

\section{Parte Experimental}

\subsection{Síntesis del CdS}

Se prepara una disolución de tioacetamida con $2.6 \mathrm{~g}$ en $100 \mathrm{~mL}$ de agua destilada en un vaso de precipitados de $250 \mathrm{~mL}$, por otro lado una disolución de cloruro de cadmio con $6.3 \mathrm{~g}$ en $200 \mathrm{~mL}$ de agua destilada. Las dos disoluciones se mezclan en un vaso de precipitados de $600 \mathrm{~mL}$ agitándose y añadiendo hidróxido de potasio en disolución hasta llegar a un $\mathrm{pH}$ de 9 para proporcionar el medio en que se lleve a cabo la reacción.

\subsection{Síntesis del ZnS}

Se prepara una disolución de sulfuro de sodio con $4.0 \mathrm{~g}$ en $100 \mathrm{~mL}$ de agua destilada en un vaso de precipitados de $250 \mathrm{~mL}$, por otro lado una disolución de cloruro de zinc con $7.0 \mathrm{~g}$ en $200 \mathrm{~mL}$ de agua destilada. Las dos disoluciones se mezclan en un vaso de precipitado de $600 \mathrm{~mL}$ agitándose y añadiendo hidróxido de potasio en disolución hasta llegar a un pH de 9 para proporcionar así el medio en que se lleva a cabo la reacción. A los compuestos sintetizados se le aplican procesos de decantado, lavado con agua destilada y secado a temperatura ambiente. 


\subsection{Caracterización}

Los semiconductores sintetizados se caracterizaron por diversas técnicas análiticas. La difracción de rayos$\mathrm{X}(\mathrm{DRX})$ se llevó a cabo en un difractómetro Siemens D5000 utilizando radiación $\mathrm{Cu} \mathrm{K} \alpha$ $1.5418 \AA$ ) la intensidad fue medida en el rango $2 \theta$ entre $5^{\circ}$ y $90^{\circ}$ con un paso de $0.05^{\circ}$. Las áreas superficiales específicas se determinaron en un aparato Quantachrome Autosorb-1 Automated Gas Sorption. Las imágenes de microscopía óptica se obtuvieron con el equipo Olympus BX60 acoplado a un software de análisis de imagen. Para la obtención de los espectros de FTIR se utilizó un espectrómetro Perkin Elmer modelo Paragon 1000PC. Los espectros de UV-Vis se realizaron en un espectrofotómetro Varian utilizando una esfera de integración.

\subsection{Proceso fotocatalítico}

Para llevar a cabo los experimentos de degradación se siguió un diseño de experimentos factorial $2^{2}$ para cada semiconductor, ver tabla 1 . Se trabajó a temperatura ambiente $\left(25^{\circ} \mathrm{C}\right)$, agitación ultrasónica de $42 \mathrm{kHz}$ de frecuencia, pH 12 y radiación ultravioleta de $365 \mathrm{~nm}$ de longitud de onda. En la tabla 2 se muestra el total de experimentos a realizar, así como las variables tomadas en cuenta para cada experimento.

Tabla 1. Diseño de experimentos.

\begin{tabular}{|c|c|c|}
\hline Nivel/Variable & Concentración (ppm) & Tiempo (h) \\
\hline Bajo & 10 & 3 \\
\hline Alto & 20 & 5 \\
\hline
\end{tabular}

Tabla 2. Experimentos de degradación.

\begin{tabular}{|c|c|c|c|}
\hline Exp & Catalizador & Concentración (ppm) & Tiempo (h) \\
\hline 1 & $\mathrm{ZnS}$ & 10 & 3 \\
\hline 2 & $\mathrm{ZnS}$ & 20 & 3 \\
\hline 3 & $\mathrm{ZnS}$ & 10 & 5 \\
\hline 4 & $\mathrm{ZnS}$ & 20 & 5 \\
\hline 5 & $\mathrm{CdS}$ & 10 & 3 \\
\hline 6 & $\mathrm{CdS}$ & 20 & 3 \\
\hline 7 & $\mathrm{CdS}$ & 10 & 5 \\
\hline 8 & $\mathrm{CdS}$ & 20 & 5 \\
\hline
\end{tabular}

Para llevar a cabo el proceso fotocatalítico se preparó una disolución de cianuro de la concentración a estudiar (10 y 20 ppm). La disolución fue preparada con un estándar de 1000 ppm y se aforó con $\mathrm{NaOH} 1.6 \mathrm{~g} / \mathrm{L}$ para dar un $\mathrm{pH}$ de 12. La disolución se vierte en un vaso de precipitado de $600 \mathrm{~mL}$ y se le agrega $150 \mathrm{mg}$ del catalizador. Se dispone homogenizar por $10 \mathrm{~min}$ en el ultrasonido, esto sin radiación ultravioleta. Después de homogenizar se toma una muestra al tiempo cero y se le hace incidir radiación ultravioleta de $365 \mathrm{~nm}$ de longitud, se toma muestra cada 30 minutos en tubos de ensayo de $13 \times 100$, estos se centrifugan por un tiempo de 10 minutos, se decantan y se depositan en un vaso de precipitado de $10 \mathrm{~mL}$ para su posterior medición. Por otro lado se preparan estándares de 5,10,15,20 y 25 ppm para realizar la curva de calibración con un electrodo de ión selectivo a cianuro. Después de realizada la curva de calibración se procede a la medición de cianuros en las muestras, determinando así el porcentaje de degradación.

\subsection{Análisis del licor madre y los sedimentos}

Después de realizadas las degradaciones se analiza el licor madre por espectroscopía de absorción atómica (AAS), a los sedimentos se les hace un análisis textural (Autosorb), análisis microtextural (microscopia óptica) y FTIR, esto con la finalidad de determinar posibles cambios en los semiconductores debido al proceso fotocatalítico empleado.

\section{Resultados y Discusión}

En la figura 1a se muestra el difractograma del CdS sintetizado donde se observa que los picos principales concuerdan con el patrón de difracción del semiconductor, también se observa ruido en el espectro, lo cual indica que el tamaño de las partículas es relativamente pequeño y que pueden existir partículas de escala nanométrica. 
En la figura $1 \mathrm{~b}$ se muestra el difractograma del $\mathrm{ZnS}$ sintetizado, en este se aprecia que sus picos principales concuerdan con el patrón de difracción del compuesto [11], también se aprecia ruido, lo cual indica que hay partículas de escala nanométrica.
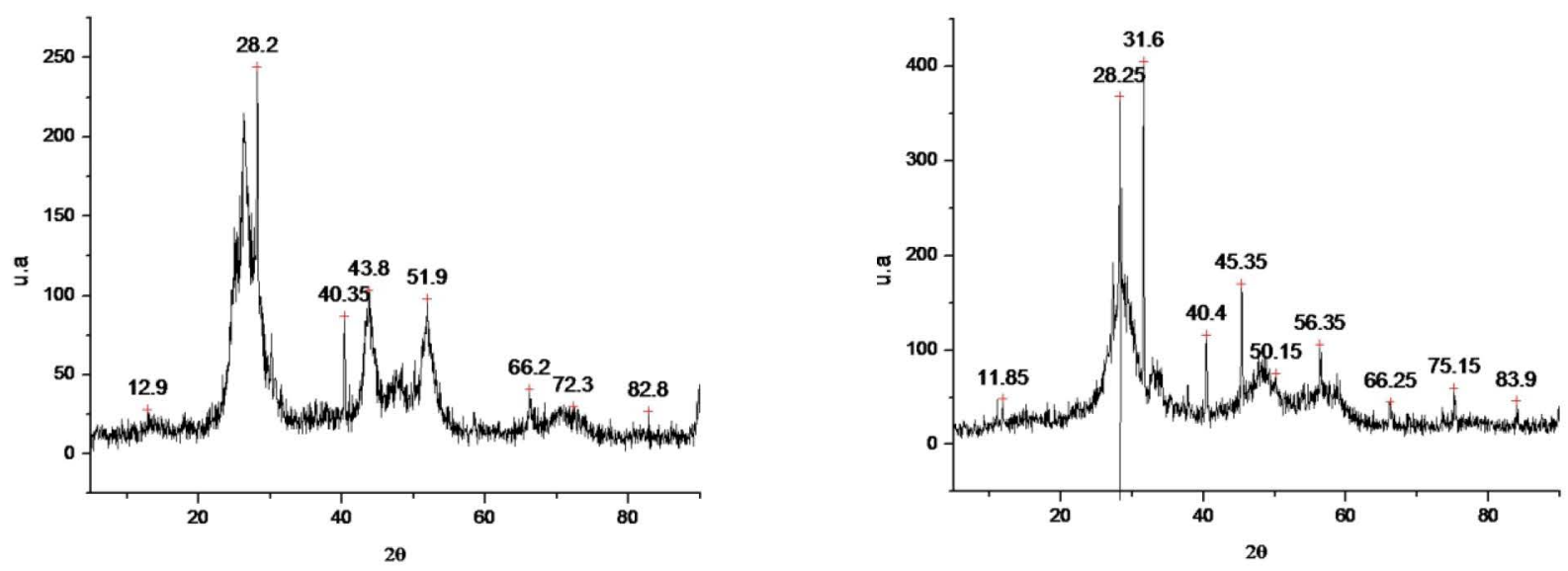

Figura 1. Difractograma de rayos- $\mathrm{X}$ del a) $\mathrm{CdS}$ y b) $\mathrm{ZnS}$.

Dado que el objetivo de este trabajo es determinar la capacidad fotocatalítica de los semiconductores antes obtenidos, se realizaron análisis de espectros de UV-Vis para determinar la energía de banda prohibida $\left(\mathrm{E}_{\mathrm{g}}\right)$ para cada uno, ya que los valores de $\mathrm{E}_{\mathrm{g}}$ deben ser menores a la energía que se aplica. En la figura 2a se muestra el espectro de UV-Vis para el $\mathrm{ZnS}$, donde se observa que el semiconductor absorbe longitudes de onda menores a $368 \mathrm{~nm}$, teniendo un valor de $\mathrm{E}_{\mathrm{g}}$ de $3.36 \mathrm{eV}$, por el contrario, en la figura $2 \mathrm{~b}$ se muestra el espectro para el CdS, apreciándose que éste absorbe a longitudes de onda menores de $573 \mathrm{~nm}$, mostrando un valor de $\mathrm{E}_{\mathrm{g}} \mathrm{de} 2.16 \mathrm{eV}$, por lo tanto ambos semiconductores pueden ser activados bajo radiación de luz ultravioleta de $365 \mathrm{~nm}$.
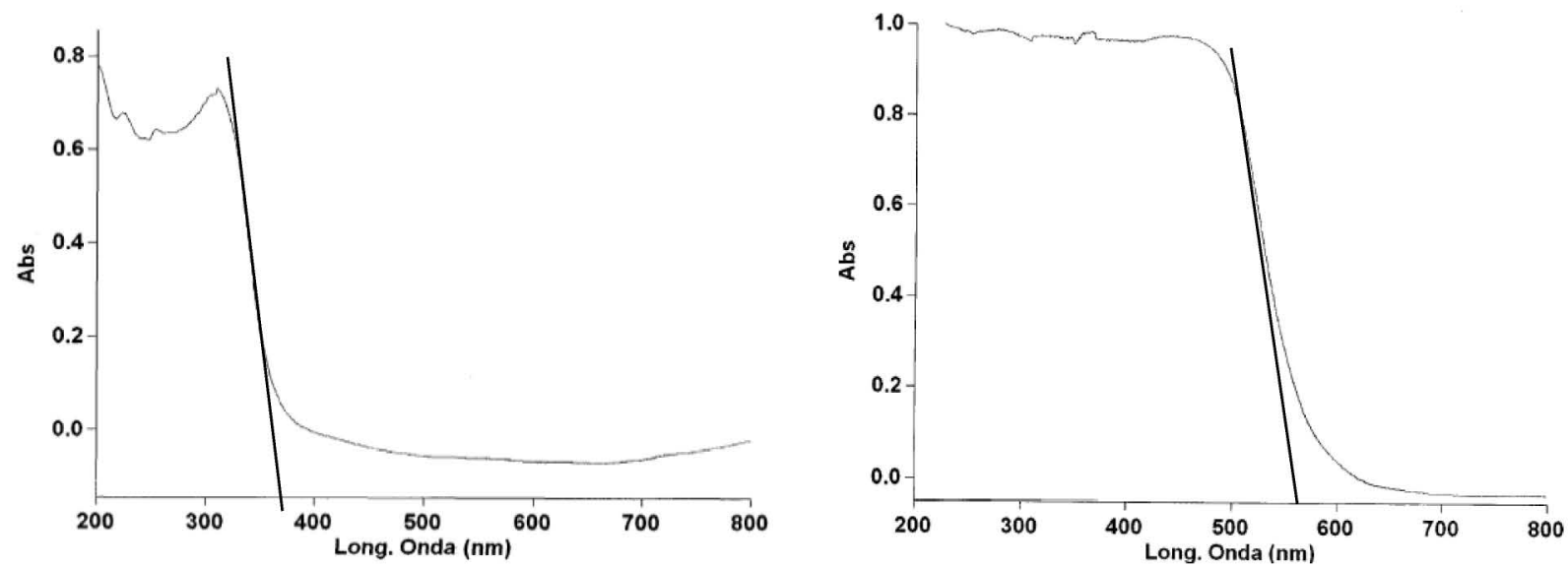

Figura 2. Espectro de UV-Vis del a) ZnS y b) CdS.

En la figura 3a se muestra la isoterma de adsorción obtenida para el CdS, obteniendo un área superficial específica de $74.75 \mathrm{~m}^{2} / \mathrm{g}$, ciclo de histérisis mostrado es angosto, lo cual indica una distribución del tamaño de poro estrecha, sin condensación capilar ni afinidad por el adsorbato. En la figura $3 \mathrm{~b}$ se muestra la isoterma de dsorción obtenida para el ZnS, con un área superficial específica de $75.17 \mathrm{~m}^{2} / \mathrm{g}$, el ciclo de histérisis es menos ngosto que para el CdS, lo cual indica que la distribución del tamaño de poro no es estrecha, muestra condensación capilar y afinidad por el adsorbato. 

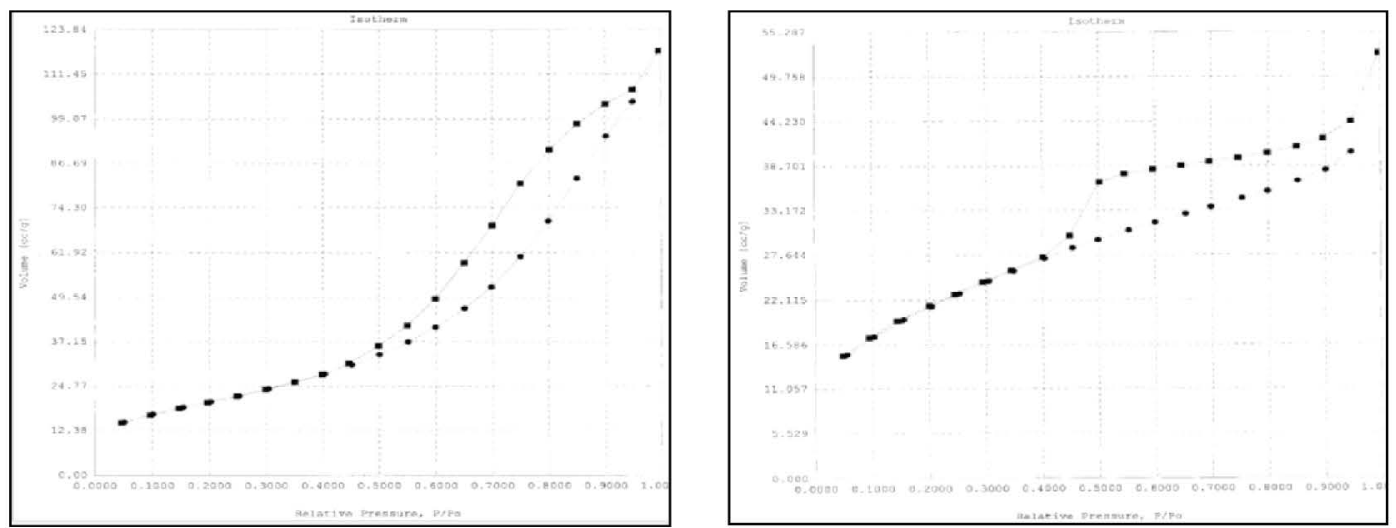

Figura 3. Isoterma de adsorción del a) CdS y b) ZnS.

Se obtuvieron micrografías de los semiconductores, en éstas se apreció tamaños de partícula disperso, siendo mayor en el $\mathrm{ZnS}$ en comparación con el CdS, esto se puede apreciar en las figuras $4 \mathrm{a}$ y $4 \mathrm{~b}$ respectivamente.
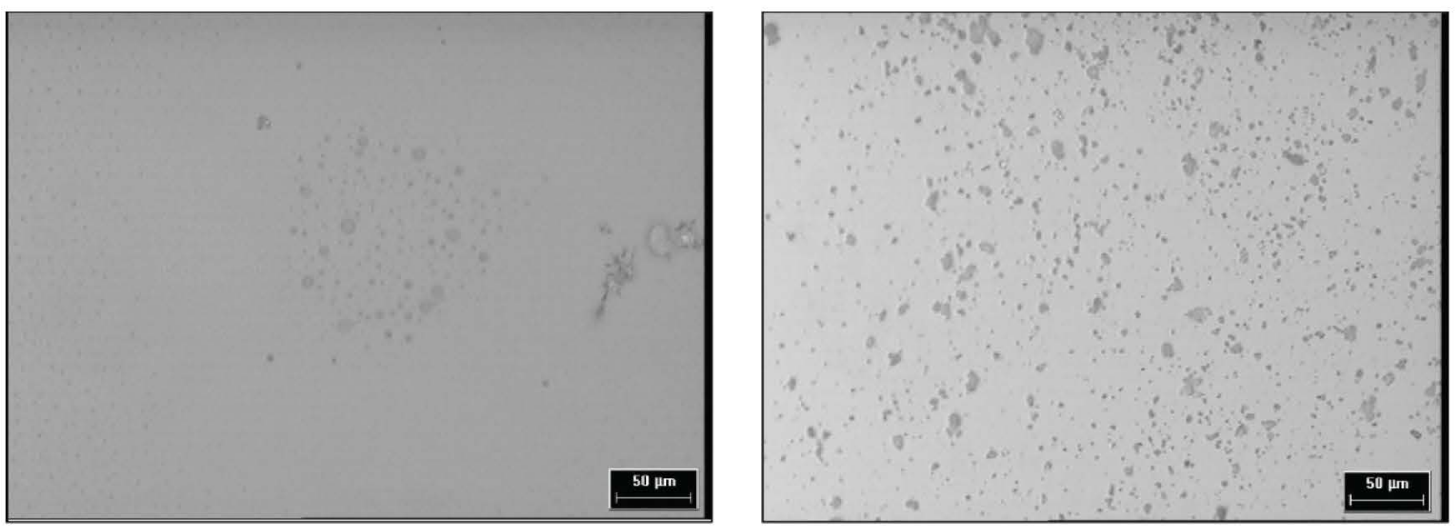

Figura 4. Micrografía del a) $\mathrm{ZnS}$ a $20 \mathrm{X}$ y b) CdS a $20 \mathrm{X}$.

Además, se comprobó mediante espectroscopia de infrarrojo (FTIR) que no quedaron precursores en los semiconductores sintetizados, lo cual se muestra en las figuras 5a y 5b, para el CdS y $\mathrm{ZnS}$ respectivamente. En estos espectros se puede apreciar la ausencia de grupos funcionales de los precursores utilizados.
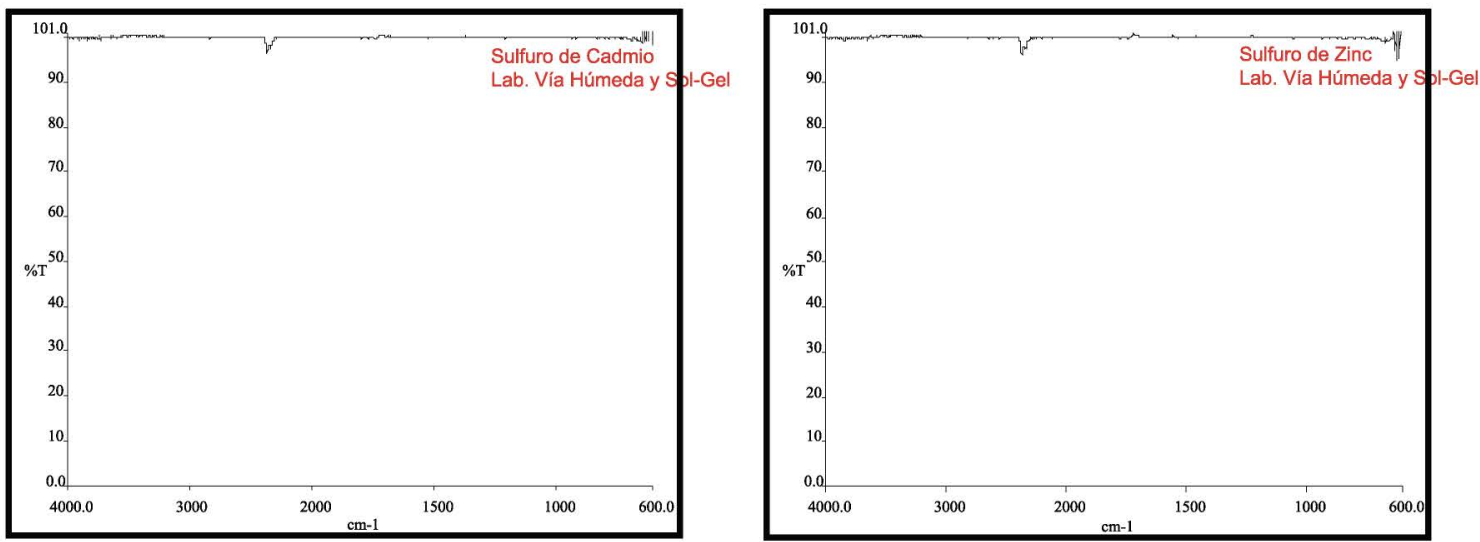

Figura 5. Espectro de FTIR para el a) CdS y b) ZnS. 
En la tabla 3 se presentan los resultados obtenidos del proceso fotocatalítico, los cuales indican que para una concentración de $20 \mathrm{ppm}$ de cianuro el CdS es el mejor fotocatalizador, pero para una concentración de $10 \mathrm{ppm}$ el CdS y ZnS compiten ya que muestran un porcentaje significativamente similar de degradación.

Tabla 3. Resultados del proceso fotocatalítico.

\begin{tabular}{|c|c|c|c|c|}
\hline No. Exp. & Catalizador & Concentración (ppm) & T (h) & Deg. \% \\
\hline 1 & ZnS & 10 & 3 & 24.38 \\
\hline 5 & $\mathrm{CdS}$ & 10 & 3 & 22.75 \\
\hline 2 & $\mathrm{ZnS}$ & 20 & 3 & 10.10 \\
\hline 6 & $\mathrm{CdS}$ & 20 & 3 & 23.88 \\
\hline 3 & $\mathrm{ZnS}$ & 10 & 5 & 34.81 \\
\hline 7 & $\mathrm{CdS}$ & 10 & 5 & 36.43 \\
\hline 4 & $\mathrm{ZnS}$ & 20 & 5 & 29.70 \\
\hline 8 & $\mathrm{CdS}$ & 20 & 5 & 50.84 \\
\hline
\end{tabular}

Para explicar el comportamiento que presentan los semiconductores en el proceso fotocatalítico llevado a cabo en este trabajo, se realizó un análisis de textura en ambos semiconductores después de ser utilizados, éstos se muestran en la figura $6 \mathrm{a}$ y $6 \mathrm{~b}$, donde se observa que el área superfícial específica disminuye considerablemente para ambos, aunque para el $\mathrm{ZnS}$ se observa una mayor disminución debido a que su afinidad por el adsorbato disminuye debido al medio del proceso fotocatalítico, lo cual hace que tienda más a aglomerarse, además, no muestra condensación capilar debido al proceso que se llevó a cabo. Para el CdS se muestra una disminución en su área superficial específica, aunque ésta no es tan drástica como lo fue para el $\mathrm{ZnS}$, esto se debe a que el medio del proceso fotocatalítico no afecta tanto al semiconductor ya que su afinidad por el adsorbato aumenta respecto al CdS sin ser utilizado en el proceso.
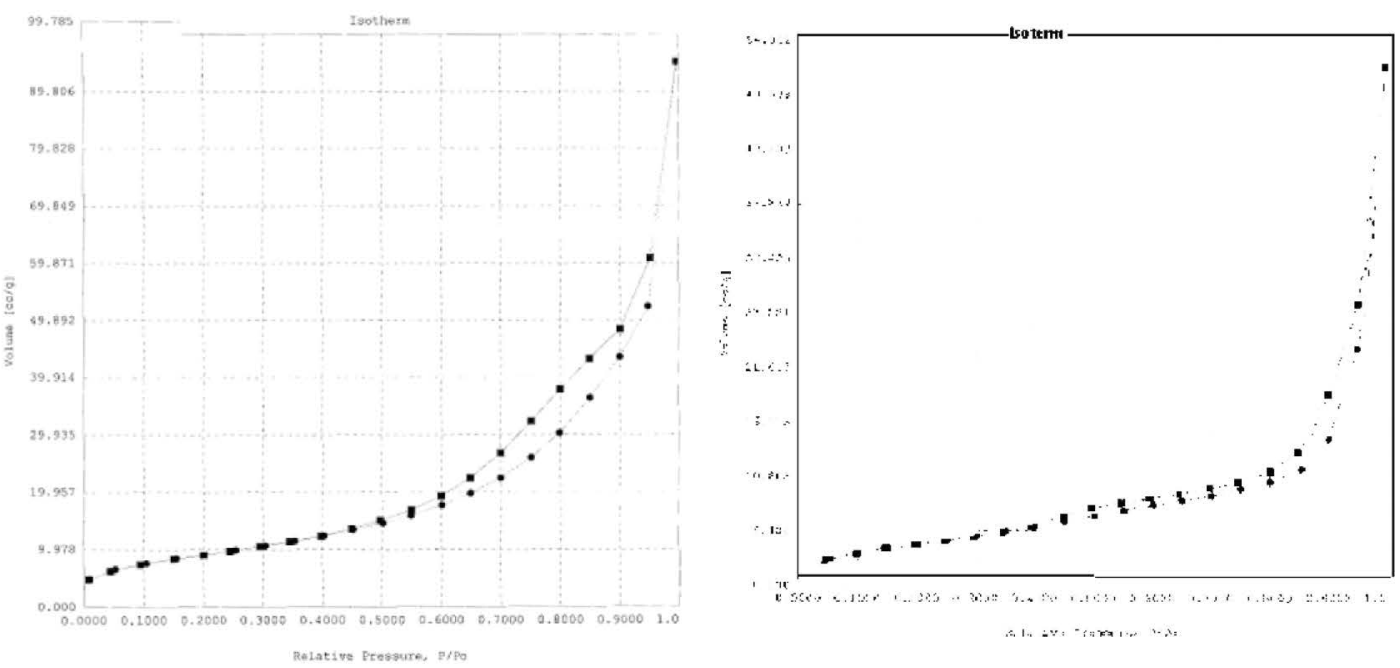

Figura 6. Isoterma de adsorción para el a) CdS utilizado y b) ZnS utilizado.

ara corroborar lo anterior se realizaron análisis por microscopía óptica. En las figuras 7a y 7b se muestran las iicrografías obtenidas del CdS y ZnS respectivamente. En éstas se pueden apreciar fenómenos de aglomeración o cual coincide con lo observado y discutido en las isotermas de adsorción. 

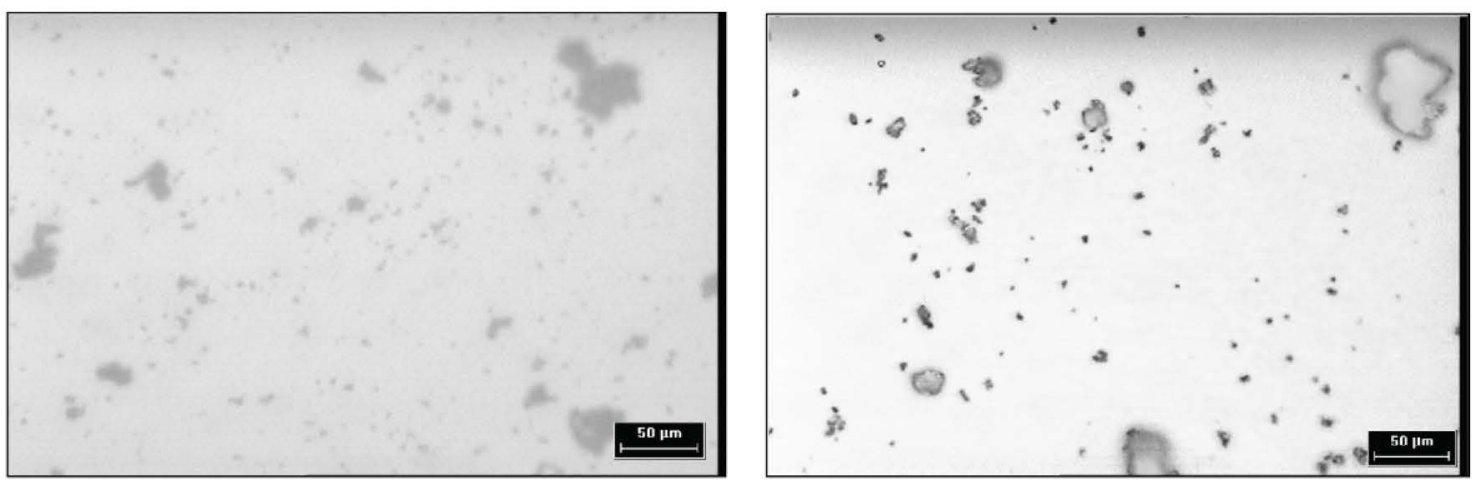

Figura 7. Micrografia óptica del a) CdS en un aumento de 20X y b) $\mathrm{ZnS}$ a $20 \mathrm{X}$.

Con la finalidad de descartar que el proceso de aglomeración se deba a la fisisorción de grupos funcionales del medio utilizado, se realizaron análisis de FTIR de los semiconductores ya utilizados, en éstos se descartó la presencia de grupos adheridos, ver figura $8 \mathrm{a}$ y $8 \mathrm{~b}$ para el $\mathrm{ZnS}$ y CdS respectivamente. También se realizó un análisis de espectroscopía de absorción atómica del licor madre con la finalidad de determinar la estabilidad de los semiconductores en el medio, encontrando una mayor inestabilidad en el $\mathrm{ZnS}$ en comparación al CdS, ya que se obtuvieron concentraciones de hasta $3.4 \mathrm{ppm}$ y $0.36 \mathrm{ppm}$ para el $\mathrm{ZnS}$ y CdS respectivamente.
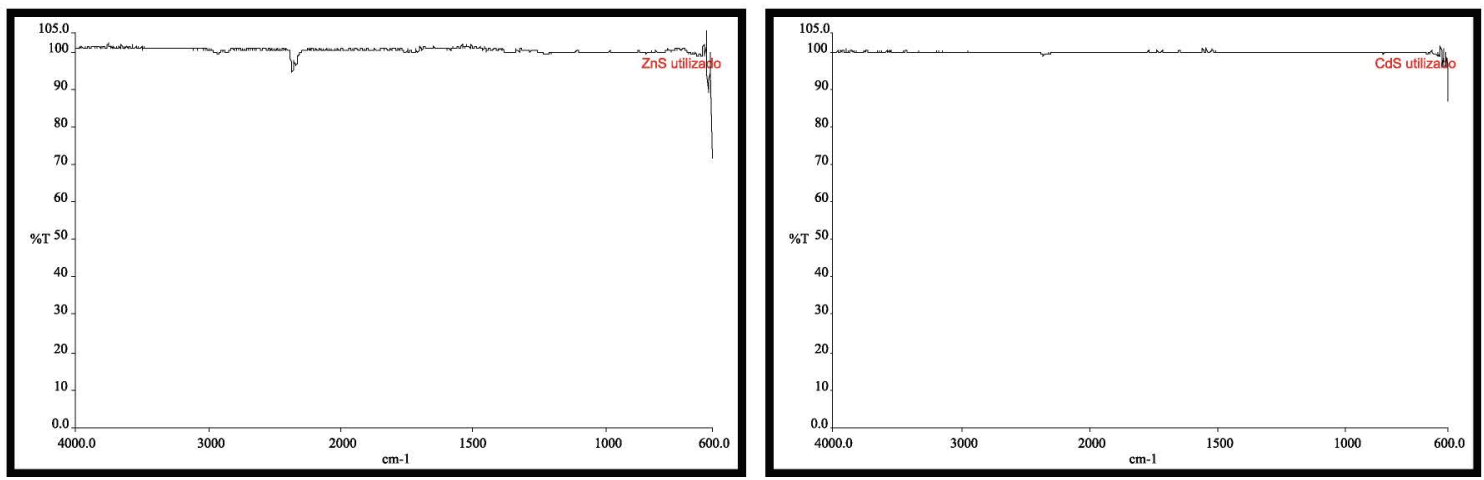

Figura 8. FTIR del a) ZnS utilizado y b) CdS utilizado

\section{Conclusiones}

A partir de los resultados se concluye que el CdS y el $\mathrm{ZnS}$ sintetizados en el laboratorio absorben a la longitud de onda de $365 \mathrm{~nm}$, donde la mayor actividad fotocatalítica la muestra el CdS a una concentración de $20 \mathrm{ppm}$ de cianuros. El ZnS se aglomera más que el CdS debido al medio del proceso. No se adhieren grupos $\mathrm{CN}^{-}$ al CdS y ZnS utilizados. El área superficial específica de ambos semiconductores cambia debido a procesos de aglomeración.

\section{Agradecimientos}

A1 PAICYT por el apoyo económico para el desarrollo de esta línea de investigación. Así como a los laboratorios de Sol-Gel y Cerámica Tradicional de la Facultad de Ciencias Químicas de la U.A.N.L.

\section{Referencias}

[1] “Water Treatment Handbook, Degrémont”, 6a Edición. Editorial Lavoiser Publishing, París (1991).

[2] O. Legrini, E. Oliveros y A. M. Braun, Chem. Rev.,93, 671-698(1993).

[3]C. P. Huang, Ch. Dong y Z. Tang, Waste Management, 13,361-377 (1993).

[4] US/EPA Handbook of Advanced Photochemical Oxidation Processes, EPA/625/R-98/004 (1998).

[5] The AOT Handbook, Calgon Carbon Oxidation Technologies, Ontario (1996).

[6] J. R. Bolton y S. R. Cater, “Aquatic and Surface Photochemistry", 467-490. G. R. Helz, R. G. Zepp y D. G. Crosby Editores. Lewis, Boca raton, FL, EEUU (1994).

[7]Zhang, F., Zhao, J., Shen, T., Hidaka, H., Pelizzetti, E., and Serpone, N., Appl. Catal. B 15, 147 (1998). 
[8] Wu, G., Wu, T., Zhao, J., Hidaka. H., and Serpone, N., Environ. Sci. Technol. 33, 2081 (1999).

[9] Lui. G., Li, X., Zhao, J., Horikoshi, S., and Hidaka, H., J. Mol. Catal. A 153, 221 (2000).

[10] J. Hodak, C Quinteros, M.I: Litter, M:I y E. San Román, J. Chem. Soc., Faraday Trans., 92, 508 (1996).

[11] Tuba Öznülüer, Ibrahim Erdogan, and Ümit Demir. Langmuir 22, 4415-4419 (2006).

[12] Henglein, A. Chem. Rev.1989, 89, 1861-1873.

[13] Hengboyin, Yujiwada, Takayukikitamura and Shozoyanagid., Environ. Sci. Technol.2001, 35,227-231.

[14] Wenfeng Shangguan and Akira Yoshida., J. Phys. Chem B 2002, 12227-12230

[15] David Malacara, Idalia Gómez y Laura García, Memorias del Congreso Nacional de Química 2005, Abril (2005).

[16] Edison Pavas, Paola Andrea Camargo, Claudia Castro y Tomás Pineda, "Oxidación Fotocatalítica de Cianuro", (2005). 